\title{
DIDÁCTICA GENERAL Y ESPECÍFICA DE LAS LENGUAS EXTRANJERAS EN CUBA, ¿AUTONOMÍA, DEPENDENCIA, COEXISTENCIA O COMPLEMENTACIÓN?
}

\section{The General and Specific Didactics of Foreign Languages in Cuba, autonomy, dependence, coexistence or complementation?}

Carlos Martínez-Linares ${ }^{1}$

carlos27mtz@gmail.com

1 Universidad de Ciencias Pedagógicas Enrique José Varona, Cuba

Fecha de recepción: 2/04/2020

Fecha de aprobación: 28/07/2020

\section{Resumen}

El presente artículo toma posición en el dilema de la asunción y contextualización de los supuestos de la didáctica general en el tratamiento de las problemáticas que acontecen en el marco de las materias específicas. En tal sentido, hemos concretado nuestro análisis al proceso docente de las lenguas extranjeras a fin de demostrar a estudiantes y colegas de nuestra área profesional la necesidad de asumir conscientemente y de manera contextualizada los aportes significativos de la didáctica general, los que, interpretados según las leyes y los principios específicos del proceso docente de las lenguas extranjeras, habrán de aportar a la solución de los problemas particulares de esa materia de estudio en el contexto pedagógico cubano actual.

Palabras adquisición segunda lengua, educación bilingüe, didáctica general, didáctica específica, lenguas extranjeras.

\section{Abstract}

Through this article, a position is assumed concerning the dilemma related to the assumption and contextualization of the fundamentals of general didactics for facing issues that take place within the environment of specific subjects. So, our analysis has focused on the field of the teaching- learning process of foreign languages to demonstrate students and colleagues of our professional area, the necessity to consciously assume significant contributions of general didactics. When interpreted according to specific didactic principles and laws, these contribute to the solution of specific problems of this subject matter within the current Cuban pedagogical context.

Keywords: bilingual education, foreign languages, general didactics, second language acquisition, specific didactics. 


\section{Introducción}

A través del presente artículo se intenta socializar ideas y reflexiones, desde una óptica interdisciplinar, en las que se describen las posiciones teóricas, metodológicas y organizativas que se asumen en torno al proceso docente de las lenguas extranjeras en el contexto educativo cubano actual. En resumen, reflexionar acerca de la incidencia de la didáctica general y en especial de sus leyes y principios en los asuntos de la didáctica específica de las lenguas extranjeras constituye el núcleo central del análisis. Para la sistematización teórica se consultaron materiales nacionales e internacionales especializados en el área de las didácticas, así como también supuestos teóricos expresados por el autor en publicaciones anteriores, todo ello debidamente referenciado. Para ello empleamos el método teórico, histórico-lógico para el análisis de las tendencias que han existido en torno a la didáctica general y su relación con la didáctica especifica en el contexto educativo cubano; de igual manera, utilizamos el análisis-síntesis para el estudio bibliográfico, la descripción y caracterización del proceso de enseñanza-aprendizaje de las lenguas extranjeras en nuestro país, así como las consideraciones de diversos especialistas referidas al diseño curricular y la dirección de dicho proceso docente. Al nivel empírico utilizamos el método del estudio documental para examinar las documentaciones rectoras o normativas que orientan el proceso de enseńanza-aprendizaje de la lengua extranjera en el sistema educativo cubano.

Las ideas planteadas en este artículo no constituyen conceptos inamovibles ni saberes hechos, con ellas se pretende estimular un análisis crítico y reflexivo para el surgir de nuevas teorías que, concebidas según las especificidades de los diversos contextos pedagógicos, contribuyan a un redimensionamiento significativo del quehacer docente. Recuérdese que en asuntos de educación no hay mejor recomendación que la que se asuma desde la perspectiva del espacio y del tiempo $y$, en consecuencia, debidamente contextualizada a las especificidades manifiestas en su salón de clases.

\section{Desarrollo}

Desde el propio surgir de la especie humana surge la necesidad de aprender el mundo, de entender una realidad que, dado su exiguo conocimiento, se convertía en una ficción, en un misterio. Tal situación constituyó una premisa no solo para la asunción del reto de enfrentar y entender lo desconocido, sino también para la difusión y aplicación de nuevas experiencias para la solución de problemas del contexto vivencial. De esta manera surge la escuela, la que considerada como un fenómeno social acogió el encargo de la transmisión de experiencias aprendidas, pero de una forma organizada e intencionada.

Fue Juan Amos Comenio (1592-1670) quien a través de su obra Didáctica Magna descifra un sistema estructurado de teorías sobre el proceso de enseñanza (Labarrere \& Valdivia, 1988). Aún en la actualidad, muchos de los supuestos teóricos aportados por Comenio sirven de soporte esencial para una dirección efectiva del proceso de enseñanza y aprendizaje en el marco de la institución escolar contemporánea. Ulteriormente aparecen otras figuras relevantes tales como Rousseau, Pestalozzi, Herbart, Disterweg, Ushinski y otros, quienes sobre la base de lo aportado por Comenio desarrollaron importantes tesis que contribuyeron al desarrollo significativo de la teoría didáctica.

En la actualidad, la didáctica puede ser admitida en sus aspectos generales y particulares. De ahí que se hable de didáctica general y didáctica específica. Mientras que la didáctica general estudia el proceso docente con un enfoque general, la específica estudia dicho proceso restringido al desarrollo de una disciplina particular (Álvarez de Zayas, 1995, p. 7). Respecto a ellas, una gran parte de la comunidad docente defiende el carácter científico de ambas, el que se fundamenta en el hecho de que poseen un objeto de estudio bien definido, propio, auténtico, un sistema de principios, leyes y regularidades, y una metodología particular para la solución de problemas relativos a la enseñanza y el aprendizaje. 
La tarea de la didáctica consiste en investigar las leyes generales de la enseñanza y el aprendizaje - en especial, de la enseñanza y el aprendizaje docente-, llevar los conocimientos adquiridos a una relación sistemática y crear con ello una base teórico-científica segura para el trabajo docente del maestro (Klimberg, 1972, p. 37).

Resulta razonable lo referido a cierta exclusividad en los objetos de estudio de las diversas disciplinas científicas de acuerdo con una determinada problemática y derivado de estos, objetivos y metodologías que se establecen para la solución real de un problema particular. Sin embargo, el hecho de ostentar incuestionables niveles de singularidad en modo alguno expresa total independencia para encarar las agravantes de los fenómenos. La complejidad siempre creciente de las realidades con las que se interactúa conduce al planteamiento de alternativas cada vez más profundas, las que concebidas bajo un carácter interdisciplinario conducen a la solución efectiva de las situaciones siempre complicadas del marco existencial actual (Martínez, 2017).

La idea anterior fundamenta la relación que se establece entre la didáctica general y la específica, a pesar de las singularidades de sus respectivos objetos de estudio.

La didáctica especial se vincula con la didáctica general, la primera revela las regularidades que se manifiestan en lo que hay de común en el proceso de enseñanza y aprendizaje de las diversas disciplinas docentes [...] Con ello, la didáctica general crea una base científica teórica sólida para el trabajo del profesor de cada materia en especial [...] Las didácticas especiales a su vez se convierten en fuentes de la didáctica general porque aportan los datos concretos de la enseñanza de cada materia (Antich, 1986, p. 15).

Tal aseveración justifica el vínculo entre ambas didácticas. Sin embargo, resulta importante acotar la imposibilidad de reducir las tareas de las didácticas específicas según lo establecido por la didáctica general. Cada disciplina deberá responder a los problemas que en torno a la enseńanza y el aprendizaje se establecen en el marco de su objeto de estudio y para lo cual no siempre la didáctica general puede responder (Labarrere \& Valdivia, 1988). Sobre esa base resulta lícito apuntar que la relación entre ambas didácticas se establece en condiciones de reciprocidad, de correspondencia y colaboración, pero con una interpretación clara del sistema categorial de la didáctica general de acuerdo con las particularidades de los diversos procesos docentes.

Precisamente, uno de los elementos que caracterizan a la didáctica específica de las lenguas extranjeras es la relación que establece con otras ciencias afines para la solución de problemas inherentes a su proceso docente, cuyo fin último es el desarrollo de la competencia comunicativa.

El desarrollo de la competencia comunicativa en lengua extranjera constituye un asunto complejo que precisa de la asunción de los aportes de otras áreas afines, como la pedagogía, la lingüística, la psicología, la didáctica general, los que, interpretados según las leyes y los principios específicos del proceso docente de las lenguas extranjeras, habrán de aportar a la solución de problemas relativos a la comunicación en idioma extranjero (Martínez, 2017, p. 73).

Sobre esa base, resulta indiscutible la significatividad de los supuestos de la didáctica general en el contexto de la didáctica específica de las lenguas extranjeras, lo que condiciona un nexo estable entre ambas didácticas en el que la primera, en su rol de base teórica metodológica con carácter universal, habrá de contribuir al cumplimiento del objetivo rector del proceso docente de las lenguas extranjeras: el desarrollo de la competencia comunicativa en lengua extranjera. El develamiento de tal relación no constituye un fenómeno nuevo; en su Didáctica Magna, Comenio dedicó varios capítulos a las didácticas específicas, dentro de ellas, refirió a la didáctica de las lenguas. Para él, la enseñanza de lenguas revestía de gran importancia por lo que en 1658 publicó una de sus más famosas obras, Janua Linguarum Reserata (Camilloni, 2007).

En la actualidad, la pedagogía cubana está sujeta a leyes y principios generales y sobre esa base orienta hacia la preparación plena del hombre para la vida; a este respecto, se declaran dos leyes:

- La relación escuela sociedad,

- La educación a través de la instrucción. 


\section{MARTÍNEZ-LINARES}

Didáctica general y específica de las Lenguas Extranjeras en Cuba, ¿̇autonomía, dependencia...

\subsection{La relación escuela sociedad}

La educación como fenómeno social habrá de condicionarse a toda una diversidad de factores que en el orden económico, político y cultural se establecen y también redimensionan, a consecuencia de los constantes cambios que de manera inobjetable, necesaria y lógica se establecen en cualquiera de los escenarios sociales. Atendiendo a esta consideración, se explica esta ley sobre la base de que la sociedad determina todo lo que tiene lugar en la enseñanza (Labarrere, 1988).

La Escuela es parte de la sociedad y formando parte de ella desempeña un papel fundamental pero subordinado a los intereses y necesidades de ella. No es, pues, el mero vínculo o relación entre la escuela y la sociedad, sino, con carácter de ley, la subordinación de la parte: la escuela, al todo: la sociedad (Alvares de Zayas, 1995, p.22).

Por consiguiente, en la perspectiva que aquí se adopta se puede confirmar que no existe sistema educacional alguno que escape a las necesidades cada vez más crecientes del contexto que lo engendra. Cumplir encomiendas educativas a partir de lo que en el espacio y momento se clama deberá marcar la pauta de lo que desde el punto de vista formativo se deba aportar.

\subsection{La educación a través la de instrucción}

Podemos definir la educación como el proceso y resultado de la formación de valores que permitirá al individuo asumir una conducta apropiada de acuerdo con las normas y principios de la moral que se establecen en un determinado contexto histórico, social y cultural. La instrucción es proceso y resultado de la asimilación consciente de conocimientos, de la formación de hábitos, así como del desarrollo de habilidades y capacidades que posibilitan al individuo transformar la realidad objetiva que lo circunda a través del trabajo creador. Como se puede apreciar, tanto la educación como la instrucción tienen personalidad propia, pero a la vez conforman un par dialéctico.
Instrucción no es lo mismo que educación: aquella se refiere al pensamiento, y esta principalmente a los sentimientos. Sin embargo, no hay una buena educación sin instrucción. Las cualidades morales suben de precio cuando están realzadas por las cualidades inteligentes (Martí, 1884, p. 375).

El proceso de enseñanza-aprendizaje en general constituye en sí mismo un proceso de formación de la personalidad del estudiante, a partir de que en el mismo se forma y desarrolla la relación entre lo cognitivo (sistema de conocimientos, hábitos y habilidades, o sea, lo instructivo) y lo afectivo (lo educativo) (González, 2009, p. 13).

Estas leyes pedagógicas aplicadas al trabajo docente se expresan en leyes de la didáctica; son las mismas para ambos procesos (Álvarez de Zayas, 1995). De la misma manera se declaran leyes de las didácticas específicas a partir de las particularidades de los respectivos objetos de estudio. Por consiguiente, pueden asumirse como base teórica orientadora con carácter obligatorio y flexible paraladirección efectiva del proceso docente de las lenguas extranjeras. Su asunción se expresa en el trabajo metodológico dirigido al desarrollo de la competencia comunicativa en idioma extranjero, la que habrá de evidenciarse en un uso correcto de la lengua extranjera para la solución de problemas de índole individual y social en el plano de situaciones comunicativas de la vida real (relación escuela-sociedad), pero también en un uso adecuado o educado de la misma, de acuerdo con la normas y patrones conductuales que resultan consustanciales al contexto sociocultural en el que se efectúa el acto de la comunicación (educación a través de la instrucción).

A modo de conclusión, podemos alegar que tales leyes marcan las pautas generales que indican la dirección del proceso docente de las lenguas extranjeras en Cuba. Las mismas están condicionadas a partir de las influencias circunstanciales y específicas de tipo político, social y educativo general, que determinan la esencia con la que tal proceso se concibe y desarrolla (Martínez, 2017). La didáctica general no está conformada solamente por leyes, sino también por principios. 
La organización interna de la clase responde de manera general a requerimientos de carácter didáctico por cuanto es la didáctica la ciencia que tiene como objeto de estudio la enseñanza y el aprendizaje como dos elementos que se desarrollan de manera paralela y responden a un sistema de leyes, principios y categorías, que son comunes para cualquier asignatura y de igual forma para la enseñanza de cualquier lengua extranjera (González, 2009, p. 17).

A juicio de Klimberg, "Los principios didácticos son aspectos generales de la estructuración del contenido organizativo-metódico de la enseñanza, los que se originan de los objetivos y de las leyes que los rigen objetivamente" (1972, p. 243). Estos, al igual que las leyes, se contextualizan a las didácticas específicas de acuerdo con las singularidades de sus respectivos objetos de estudio. De acuerdo con ello, se reconocen como patrones generales para la dirección de cualquiera de los procesos docentes y que conformando un sistema, constituyen la expresión de las leyes de la propia didáctica general. Existen diversas formas en las que estos se plantean (Danilov \& Skatkin, 1980; Klein, 1959; Klimberg, 1972; Labarrere \& Valdivia, 1988; Savin, 1976; Tomaschewsky, 1966; Ushinski, 1957).

A pesar de la diversidad de propuestas, existe consenso al expresarse que los principios de la didáctica general fundan las regularidades generales y esenciales que rigen la dirección científica de cualquiera de los procesos docentes (Martínez, 2017). Se conciben, además, como fuentes de indicaciones didácticas generales que resultan válidas para cualquiera de las áreas del ejercicio docente. Sirva tal postulado para fundamentar el nexo que se establece entre la didáctica general y las específicas.

En Cuba, al asumirse como fundamento para la educación el enfoque histórico-cultural, se asume la propuesta de Labarrere y Valdivia (1988):

- Principio del carácter educativo de la enseńanza.

- Principio del carácter científico de la enseñanza.

- Principio de la asequibilidad.
- Principio de la sistematización de la enseñanza.

- Principio de la relación entre la teoría y la práctica.

- Principio del carácter consciente y activo de los alumnos bajo la guía del profesor.

- Principio de la solidez en la asimilación de los conocimientos, habilidades y hábitos.

- Principio de la atención a las diferencias individuales.

- Principió del carácter audiovisual de la enseńanza: unión de lo concreto y lo abstracto.

La didáctica cubana de las lenguas extranjeras asume los principios aportados por Labarrere y Valdivia y, al igual que las leyes generales de la didáctica, los interpreta a partir de las especificidades de su objeto de estudio. Sobre esa base se ofrece a continuación una explicación resumida, así como su interpretación según las particularidades del proceso docente de las lenguas extranjeras en Cuba.

\subsection{Principio del carácter educativo de la enseńanza}

Reafirmamos la tesis de que la educación constituye un fenómeno social destinado al desarrollo integral de la personalidad del individuo. Tal intención habrá de evidenciarse en un desarrollo cognitivo, pero también axiológico que permita al individuo participar en la trasformación de la realidad que lo circunda de una manera efectiva, así como educada de acuerdo con las normas y principios que rigen la conducta ciudadana en un determinado contexto histórico, social y cultural.

El principio del carácter educativo de la enseñanza, contextualizado al objeto de estudio de la didáctica de las lenguas extranjeras, reviste de una gran importancia al asumirse como aspiración suprema de su proceso docente el desarrollo de la competencia comunicativa. La misma es definida por Hymes en 1972 como el conocimiento de la lengua y uso ( Richards, 2013). De acuerdo con ese precepto, y en relación con el principio del carácter educativo de la enseńanza en el proceso docente de las lenguas extranjeras, se consideran dos dimensiones claves que integran el concepto de competencia comunicativa: 
la dimensión sociolingüística y la sociocultural. Lo sociolingüístico refiere a la habilidad de comunicarse de acuerdo con el contexto social en el que se desarrolla el proceso de comunicación, mientras que la dimensión sociocultural se explica en la habilidad de asumir una conducta que se ajuste al espacio cultural en el que se establece la comunicación. Esta incluye, entre otros elementos, la relación espacial y de contacto entre las personas en una conversación, la gesticulación, así como las formas en que se actúa en situaciones sociales similares en una cultura y la otra (Font, 2006).

En virtud de lo planteado, asevérese que en el proceso docente de las lenguas extranjeras resulta trascendental la realización de un trabajo didáctico que se enmarque no solo en el estudio y uso funcional de los recursos lingüísticos para una correcta interacción comunicativa, sino también en la consideración de aquellos elementos que con carácter social e intercultural habrán de garantizar un apropiado proceso de la comunicación en lengua extranjera.

\subsection{Principio del carácter científico de la enseńanza}

Resulta acertado afirmar que la complejidad de la realidad objetiva en la que vivimos repercute en cada una de las esferas que integran cualquier sociedad, en la que se incluye la escuela como fenómeno social. Ante tal complejidad, surge la necesidad de nuevas soluciones que, fundamentadas desde la óptica de la ciencia, coadyuven a la solución de problemas complejos que derivan de la lógica de desarrollo que opera en el mundo de hoy. En tal sentido, el principio de la cientificidad orienta hacia la realización de un proceso docente que habrá de diseñarse asumiéndose como fundamento esencial los avances de la ciencia y en particular los resultados de la investigación educacional. Lo anterior se interpreta en un redimensionamiento constante de la concepción curricular y de la dirección de los procesos docentes, de acuerdo con la lógica del método científico, lo que presupone la asunción de nuevos enfoques y métodos, así como medios de enseńanza y aprendizaje que derivan de la actividad científica de especialistas y profesores del campo de las ciencias pedagógicas, de las lenguas extranjeras y otras áreas afines.

En la actualidad, la filosofía dialéctico-materialista constituye el fundamento científico-metodológico esencial del proceso docente de las lenguas extranjeras en Cuba, por lo que se desarrolla bajo una orientación histórico-cultural, asumiéndose la idea de la zona de desarrollo próximo aportada en 1974 por la escuela histórico-cultural del psicólogo soviético Lev S. Vigotsky.

Varios han sido los métodos y enfoques asumidos en Cuba para la enseñanza de las lenguas extranjeras. Durante las décadas de los 70 y 80 se asumió el método consciente-práctico, propuesto por el psicólogo soviético B.V. Belyaev, el que a criterio de Corona (1988) resulta significativo en cuanto "promueve la apropiación reflexiva y gradual de la lengua extranjera (formación de hábitos y habilidades) y de su formación sociocultural, enfatiza en la actividad práctica, y atiende al nexo con la lengua y la cultura materna" (p. 57). Según Góngora (2014a), a la luz del enfoque comunicativo para la enseñanza de lenguas extranjeras, la concepción del método consciente-práctico fue redimensionada en Cuba, apareciendo una nueva variante: el método consciente práctico con enfoque comunicativo. Sobre esa base en el contexto actual del proceso docente de las lenguas extranjeras en Cuba se establece como concepción metodológica el enfoque comunicativo con elementos del método consciente-práctico en tránsito hacia el enfoque comunicativo basado en tareas. Según Arroyo, esta concepción metodológica

[...] refleja el carácter activo del aprendizaje tanto en la adquisición de conocimientos como de habilidades, prestando atención a la precisión de la fluidez enmarcada en situaciones reales de comunicación signadas por el uso de funciones comunicativas (citado en Cabrera, 2004, p. 83).

Según Cabrera (como se citó en Góngora, 2014b), dicha concepción metodológica habrá de caracterizarse de la siguiente manera:

- La célula principal del proceso docente la conforma la tarea, la que tiene como fin las necesidades comunicativas de los estudiantes. 
- Utiliza la resolución de tareas, favoreciendo tanto su práctica como el uso de estrategias.

- Propicia la evaluación conjunta y valora el progreso tanto individual como grupal.

- Pondera la solución de problemas que impliquen el desarrollo de capacidades, como las siguientes: resumir, inferir, deducir, generalizar, transferir.

- Incentiva la constante reflexión sobre el aprendizaje.

- Concibe el creciente nivel de complejidad en las tareas.

- Concibe las etapas para la formación de hábitos y desarrollo de habilidades: reconocimiento, fijación, consolidación, producción controlada, producción libre.

Por lógica, la asunción de nuevos enfoques exige nuevos fundamentos teóricos que sustenten la estructura curricular que se diseñe (Martínez, 2017). En tal sentido, la concepción curricular que se asume en el proceso docente de las lenguas extranjeras en Cuba se concibe según la concepción metodológica antes mencionada.

Diversos autores han investigado el diseño curricular de programas para el proceso docente de lenguas extranjeras. Entre ellos se destacan Nunan (1993), Breen (1997) y Richards (2013). De singular importancia es la propuesta de Richards (2013), quien plantea tres enfoques diferenciados en el orden de los componentes que los integran: el centrado en el contenido (forward), el centrado en el proceso (central) y el centrado en los resultados (backward).

El enfoque central considera al contenido como componente base para la formulación de los objetivos y del resto de los componentes que ensamblan el programa de estudio. Este prepondera en el diseño de programas formales orientados al estudio de las estructuras lingüísticas y a la ejercitación, tales como el gramatical y el audiolingual. Dicho enfoque fundamenta, además, el diseño de los programas nocionales funcionales así como el de los que se sustentan en los enfoques de la instrucción basada en el contenido (Content-Based Instruction) y del aprendizaje integrado del contenido y la lengua (Content and Language Integrated Learning).

Respecto del enfoque centrado en el proceso, se puede enunciar que en él, lo metodológico ocupa un lugar inicial. Las técnicas, los métodos y los procedimientos determinan el estado del resto de los componentes curriculares. Tal concepción se aplica en el diseño de los programas de aprendizaje mediante tareas y los programas procesales. Los primeros se basan en la planificación de tareas comunicativas en torno a un tema y no a un objetivo metodológico, tal y como se sugiere en los modelos de Breen (1997). El contenido en este tipo de programa es el propio proceso de aprendizaje, en el que el estudiante como centro del proceso docente determina sus estrategias de aprendizaje a partir de su propio estilo. Los programas procesales proponen un banco de actividades basadas en situaciones reales y según las necesidades e intereses de los estudiantes. Ejemplos de programas procesales son los basados en el enfoque natural de Krashen y Terrel. Las técnicas recomendadas por estos se basan en métodos diseñados y adaptados a los principios del enfoque natural, por lo que proponen el uso de actividades propias del método de respuesta física total, del método directo, así como del enfoque comunicativo.

El tercer enfoque propuesto por Richard parte de la consideración del objetivo como componente rector y del cual deriva el planteamiento del resto de los componentes que integran el proceso docente. El mencionado autor califica este enfoque como una tradición bien establecida en el contexto de la educación general y plantea, además, que en los últimos ańos resurge como un enfoque prominente en el proceso de diseńo curricular para la enseñanza y el aprendizaje de la lengua extranjera. Este tercer enfoque se expresa en el diseño de programas sustentados en la versión 2 del enfoque para la enseñanza de lengua basada en tarea, así como en la educación basada en competencia (Competency Based Education).

En Cuba, a partir de la concepción metodológica que se orienta para el proceso docente de las lenguas 
extranjeras, se asume este último enfoque para el diseño de los programas de estudio. Todo ello, avalado por el estudio y la actividad científica desplegada por nuestros profesores.

\subsection{Principio de la asequibilidad}

Se considera al principio de la asequibilidad como uno de los más antiguos en la historia de la didáctica (Labarrere \& Valdivia, 1988). El mismo "toma como base la relación entre las exigencias de rendimiento que el maestro plantea y la actual capacidad de rendimiento del alumno" (Klimberg, 1972, p. 255). De manera más concreta y refiriéndose a él, Comenio explica que "al estudiante se le debe presentar, primero lo que está más cerca de él; sucesivamente, lo que le sigue inmediatamente, lo que está un poco más lejos, lo que está mucho más lejos y, por último, lo más distante" (citado en Jiménez \& Falcón, 2006, p. 18).

Tal idea se asemeja a la teoría de la zona de desarrollo próximo del psicólogo soviético Lev S. Vigotsky, la que constituye uno de los fundamentos esenciales del proceso docente de las lenguas extranjeras en Cuba (Martínez, 2017). Tal teoría se precisa de la siguiente manera:

La distancia que media entre los planos intrapsicológico (desarrollo actual) e interpsicológico (desarrollo potencial), o sea, la distancia entre el nivel real de desarrollo, determinado por la capacidad de resolver independientemente un problema y el nivel de desarrollo potencial, dado en la posibilidad de solucionar un problema bajo la guía de un adulto o bajo la colaboración de otro compañero más capaz (Pulido, 2005, p. 50).

El principio de la asequibilidad interpretado desde el ámbito de la didáctica específica de las lenguas extranjeras significa el planteamiento de objetivos, contenido, métodos, así como del resto de los componentes que integran el proceso docente, a partir del desarrollo real que se expresa en el estudiante, los que se irán complejizando según las necesidades y potencialidades evidenciadas en el proceso de interacción que con el objeto de estudio se establece bajo la guía del profesor. De la misma manera, se irá redimensionando el rol del profesor y del estudiante en la medida que se transite por las diferentes etapas del ciclo de enseñanza y aprendizaje. Tal orientación se explica de la siguiente forma. Se inicia con una etapa de reconocimiento de los elementos formales de la lengua, luego se transita por una etapa de entrenamiento comunicativo controlado a través de la realización de ejercicios para la internalización de los elementos formales de la lengua, luego a una etapa semicontrolada con el uso de actividades pseudocomunicativas que posibiliten un mayor nivel de internalización de la forma de la lengua, pero intencionado, además, al reconocimiento del significado de los aspectos lingüísticos en el discurso comunicativo. Se culmina con una etapa de práctica comunicativa libre en la que el desarrollo alcanzado por el estudiante habrá de evidenciarse en la habilidad de expresarse de forma fluida, espontánea y libre, en situaciones comunicativas cercanas a la realidad. Todo ello opera de forma gradual, transitándose de lo simple a lo complejo para garantizar los niveles de asequibilidad posible del contenido.

\subsection{Principio de la sistematización de la enseñanza}

El carácter sistémico constituye una característica básica de cualquiera de los procesos docentes, en el que se establece una relación de tipo subordinada e integradora entre los componentes y eslabones que lo conforman para el cumplimiento de un único fin.

El aprendizaje se desprende de todo un proceso en el que de manera intencional y sistémica se organizan sus partes, y se conforma un todo, que habrá de resultar significativo, en tanto en él se establezcan las condiciones idóneas para cumplimentar su objetivo (Martínez, 2007, p. 81).

La afirmación anterior justifica la consideración de las siguientes tareas didácticas para la implementación de este principio (Klimberg, 1972):

- De lo próximo a lo distante.

- De lo fácil a lo difícil.

- De lo conocido a lo desconocido.

- De lo individual a lo general. 


\section{Revista Caribeña de Investigación Educativa | 2021, 5(1), 148-161}

- De lo concreto lo abstracto.

La sistematización en el proceso docente de la lengua extranjera radica en lo siguiente (Antich, 1986):

- La existencia de objetivos específicos para cada nivel, curso, unidad, clase y actividad.

- El desarrollo escalonado de capacidades y habilidades en cada etapa, respondiendo a los objetivos formulados.

- La articulación entre los niveles, los cursos y las unidades de lo simple a lo complejo, de lo conocido a lo desconocido y de lo concreto a lo abstracto.

- La progresión cíclica en la ejercitación, la cual, partiendo de la imitación oral, evoluciona en cada clase o serie de clases hacia la reproducción oral y escrita, y culmina en la aplicación, también oral y escrita, de las adquisiciones en situaciones nuevas.

- La comprobación sistemática de conocimientos y habilidades.

- La interrelación sistemática y planificada entre contenidos y actividades, y la función educativa de la enseñanza de idiomas.

Consecuentemente con lo referido al principio de la asequibilidad, y con el claro propósito de fundamentar la implicación del principio de la sistematización en nuestra didáctica específica, sostenemos la idea de que "la enseñanza y el aprendizaje de las lenguas extranjeras es, por su esencia, un proceso sistémico, integrado por etapas de desarrollo (ciclo de aprendizaje), para el logro de un desarrollo gradual del pensamiento y del actuar comunicativo de los estudiantes” (Martínez, 2017).

\subsection{Principio de la relación entre la teoría y la práctica}

Para el análisis de este principio comencemos planteando que el mundo es cognoscible, que los fenómenos que acontecen en la realidad objetiva pueden ser conocidos y que la solución de sus problemas solo se logra a través de la actividad. Precisamente es a través de esta que se verifica, modifica y perfecciona la teoría aprendida. Tal idea corrobora el carácter científico de la enseñanza.

[...] la enseñanza vinculada a la vida significa dar respuestas a las cuestiones del marco vivencial humano, las que solo se comprueban en la práctica, en el accionar cotidiano, donde lo aprendido de forma teórica se redimensiona, se perfecciona y se hace verdadero como resultado de su aplicación consciente en la solución de problemas propios de la vida (Martínez \& Novo, 2007, p. 82).

Conforme con la idea anterior, y en consonancia con los enfoques contemporáneos para el proceso docente de las lenguas extranjeras, se puede afirmar que la esencia de este último radica no solo en el conocimiento teórico de la lengua, sino también y por sobre todo, en el uso consciente de esta para la solución de problemas relativos a la comunicación. En correspondencia con ello, la condición primordial para la enseñanza y el aprendizaje de las lenguas extranjeras radica en la consideración de la práctica como única vía para la internalización de los elementos estructurales de la lengua, y su uso de acuerdo con el significado que adquiere en el discurso. Por tanto, el conocimiento y uso de la lengua extranjera como medio de comunicación se logra, se verifica y se perfecciona solo en situaciones reales de práctica comunicativa.

La consideración del principio de la vinculación de la teoría con la práctica se evidencia en un proceso docente que sostiene como postulado fundamental la preparación para la vida del estudiante, la que como resultado del conocimiento (saber) y el desarrollo de la habilidad (hacer) habrá de expresarse en la capacidad de participar, de forma consciente, correcta y apropiada, en el proceso de la comunicación en lengua extranjera (Martínez, 2007, p. 82).

El principio de la vinculación de la teoría con la práctica predomina durante todo el proceso docente de la lengua extranjera a través de dos etapas fundamentales: la cognitiva (fase de presentación, práctica controlada, semicontrolada), en la que el estudiante adquiere los conocimientos lingüísticos y las habilidades, y una segunda denominada etapa comunicativa (fase libre), en la que sobre la base de situaciones 
comunicativas cercanas a la realidad, se adquiere un desarrollo de la competencia comunicativa a través de la práctica.

\subsection{Principio del carácter consciente y activo del alumno bajo la guía del profesor}

En el análisis que hasta el momento hemos realizado ha quedado claro el propósito del proceso educativo cubano, explicado en una formación integral en el individuo que le permita de una manera consciente, eficiente y equilibrada interactuar con el objeto de trabajo a fin de satisfacer en un máximo nivel las necesidades cada vez más crecientes del contexto social al cual se pertenece y debe.

En todo este proceso de formación habrá de esclarecerse el papel de cada uno de los componentes personales que participan en el proceso docente, a saber, el profesor y el estudiante. Al primero le corresponde la dirección del proceso docente, pero considerando al estudiante como centro de la labor educativa que se despliega. Sobre esa base habrá de diseñarse y aplicarse una estrategia de trabajo, la que fundamentada en los resultados de un diagnóstico de la realidad pedagógica, habrá de conducir a la consumación de un proceso docente en el que de manera gradual se redimensionen los roles de los participantes, manifestado en un mayor nivel de protagonismo estudiantil frente a una disminución paulatina del papel protagónico del docente. Todo ello, a la par del desarrollo cognitivo y axiológico que de forma consciente y palpable en el discípulo se manifieste.

La concepción metodológica del proceso docente de las lenguas extranjeras en Cuba requiere de la consideración de este principio, de ahí que en cada etapa que conforma el ciclo de aprendizaje se defina de forma clara su objetivo, el foco de atención, la técnica a utilizar, así como el rol del profesor y del estudiante. En todo este trabajo se sostiene la idea de que "debido a la propia naturaleza del lenguaje y su relación con el pensamiento, la participación activa y consciente del alumno en la clase de lengua extranjera constituye el eje de su aprendizaje" (Antich, 1986).
De tal manera, habrán de crearse las condiciones que le permitan al estudiante responsabilizarse de manera conciente, responsable con su propio aprendizaje. Lo anterior habrá de evidenciarse en un desarrollo de la competencia comunicativa, distinguida en un uso conciente y correcto de los elementos formales de la lengua en función de la comunicación; claridad en el empleo adecuado de las formas verbales y no verbales de la comunicación, en una expresión lógica en el discurso tanto oral como escrito, así como en una participación adecuada en el acto de la comunicación, de acuerdo con las circunstancias de tipo social y cultural.

\subsection{Principio de la atención a las diferencias individuales}

Este principio reconoce la necesidad de instruir y educar a los estudiantes en el colectivo y para el colectivo, sin perder de vista la atención a sus diferencias individuales (Labarrere \& Valdivia, 2009, p. 68). A nuestro juicio, las diferencias individuales se definen como el conjunto de características personales o rasgos de la personalidad que se manifiestan en la cotidianeidad del pensar y del hacer. Extrapolada tal idea al ámbito de la instrucción, resulta medular la identificación de las particularidades que tipifican la realidad individual de cada estudiante. Ello resulta imprescindible para el logro de los objetivos que se establecen.

En asuntos de instrucción, no hay mejor recomendación que la que se asuma desde la perspectiva del espacio y del tiempo, pero debidamente contextualizada a las especificidades que de manera grupal, pero también individual, se manifiestan en el salón de clases (Martínez, 2007, p. 82).

La realización del principio de la atención a las diferencias individuales en el proceso docente de las lenguas extranjeras significa considerar al grupo en su integridad, pero poniendo especial atención a la multiplicidad de formas en las que se expresa la personalidad de cada uno de sus integrantes. Por ello, habrán de concebirse aquellos factores de tipo cognoscitivo, sociológico, psicológico, ideológico, físico, cultural, económico, y otros, que inciden en el proceso de aprendizaje de la lengua extranjera. 


\section{Revista Caribeña de Investigación Educativa | 2021, 5(1), 148-161}

Sobre esa base se apoya la necesidad de realizar un diagnóstico integral, el que asumido como un proceso con carácter instrumental, brinde la posibilidad de discernir en cuanto a necesidades, carencias, potencialidades y estilo de aprender de cada educando. Todo ello dará la posibilidad de transformar de forma gradual y significativa el estado real de cada estudiante. En tal sentido, se redimensiona el papel del profesor de lenguas extranjeras en el diseño e implementación de técnicas de trabajo docente, enmarcadas en el trabajo cooperativo, pero también en el trabajo individual, en el que los diferentes estilos de aprendizaje se tienen en consideración. Para ello se indica la creación de métodos, medios y formas de enseńanza de carácter inclusivo en condiciones de diversidad escolar.

A modo de colofón, plantéese que la consideración de este principio de la didáctica general en el ámbito de la enseñanza y el aprendizaje de las lenguas extranjeras habrá de garantizar la ejecución de un proceso docente en el que, considerándose la heterogeneidad manifiesta en él, habrá de contribuir al logro de un objetivo de interés común: el desarrollo de la competencia comunicativa.

\subsection{Principio de la solidez de los conocimientos, los hábitos y las habilidades}

Este principio exige del docente la ejecución de una actividad docente caracterizada por su carácter sistemático, a fin de garantizar una asimilación consciente, perdurable y significativa del contenido. A partir de lo anterior, este principio exige un tipo de enseñanza que garantice el desarrollo de una memoria a largo plazo, la que solo se logra a través de una interacción con el objeto de estudio que transite por las etapas del reconocimiento, la práctica reproductiva, productiva y finalmente la creativa en situaciones muy cercanas a la vida real. En concordancia con la tesis anterior, veamos la siguiente idea:

No solo la "motivación" interesante, la exposición de un problema, la narración del maestro que despierta el entusiasmo y la viva conversación en la clase, forman parte de la enseñanza. Integrantes de la enseñanza son también, la repetición y sistematización planificada, la práctica intensiva y la aplicación variada de los conocimientos y las capacidades (Klimberg, 1972, p. 260).

Para el proceso docente de las lenguas extranjeras, resulta de extrema importancia el desarrollo de los procesos de la memoria, expresada en una internalización real del contenido lingüístico para un uso consciente del mismo durante el proceso de la comunicación. A partir de ahí, muchos han sido los intentos para garantizar lo anteriormente expresado a través de la propuesta de ciclos de aprendizajes para la enseńanza y el aprendizaje de la lengua extranjera, entre ellas se destacan las aportaciones de Dakin (1973), Byrne (1976), Johnson (1982), Hammerly (1982), Nunan (1989), Crookes y Chaudron (1991), Penny Ur (1996).

En Cuba, durante muchos años se ha asumido el ciclo de aprendizaje de la lengua extranjera PPP propuesto por Byrne. A través de él, se realiza un entrenamiento sistémico y sistemático estructurado por las siguientes etapas: la orientación y la motivación del nuevo contenido lingüístico (etapa de presentación y práctica inicial), la asimilación, el dominio y la sistematización del contenido (etapas de práctica controlada, semicontrolada y libre) y, finalmente, la etapa de práctica comunicativa libre. Esta preparación sostiene como núcleo los ejercicios, las actividades pseudocomunicativas y las tareas comunicativas.

A pesar de la preferencia a favor del ciclo de aprendizaje de Byrne en Cuba, se intenta la implementación de una nueva idea sugerida por Font (2006), tendiente a la solución de problemas relativos a la comunicación en lengua extranjera bajo la perspectiva del enfoque comunicativo. Tal propuesta responde a la concepción metodológica para las lenguas extranjeras en Cuba: el enfoque comunicativo con elementos del método consciente-práctico en tránsito hacia el enfoque comunicativo basado en tareas. Las fases del ciclo son las siguientes:

- Práctica comunicativa problémica inicial.

- Determinación, análisis y solución del problema docente. 
- Práctica controlada dirigida a la forma.

- Práctica guiada.

- Práctica libre integradora.

- Aplicación creativa.

A modo de conclusión se puede alegar que este principio resulta primordial para el logro efectivo del proceso de enseńanza y aprendizaje de la lengua extranjera. Corresponde pues al docente crear las condiciones idóneas que posibiliten al estudiante la práctica necesaria, en la que el papel protagonista del docente disminuye en la misma medida en que el estudiante transitando por los diferentes niveles de la práctica internalice de manera consciente el contenido, se apropie de las vías de trabajo y las haga suyas, en la medida que las transforme para solucionar de manera independiente y creativa problemas relativos a la comunicación en lengua extranjera.

\subsection{Principio del carácter audiovisual de la enseńanza: unión de lo concreto y lo abstracto}

Comenius planteó la necesidad de la experiencia sensorial antes de la explicación verbal de las cosas, por lo que ponderó el uso de la observación como método y el empleo de láminas. Sobre esa base estableció los principios del método intuitivo (Konstantinov \& al., 1977, p. 23). De esta manera propuso su propio método para la enseńanza de las lenguas extranjeras, aplicándose por primera vez el uso de la visualización a través del uso de medios de enseñanza (Antich, 1986).

Es necesario que el conocimiento empiece por los sentidos (cierto es que nada hay en el entendimiento que antes no haya estado en el sentido). ¿Por qué, pues, ha de darse comienzo a la enseñanza por la narración verbal y no mediante la inspección de la cosa? Después, una vez presentada la cosa, venga la narración para explicar más profundamente lo expuesto (citado por Agramonte \& al., 2012).

Pestalozzi fue otro personaje que también refirió a la intuición. Según su criterio, esta constituye el punto de partida del conocimiento. Desde este punto de vista sostiene que el ver resulta premisa indispensable para entender la esencia de los fenómenos, plantea que primero se necesita conocer la forma de los objetos (geometría, dibujo, escritura) para luego adquirir una interpretación real de los mismos.

Desde una concepción más actual se afirma que en el proceso de la intuición participan todos los órganos de los sentidos del ser humano, los que en pleno funcionamiento nos ponen en contacto con la realidad objetiva circundante. Tal idea extrapolada al campo de la instrucción justifica el uso de los medios de enseñanza audiovisuales para el logro de un proceso de aprendizaje que transite según el precepto de la unidad de lo concreto y lo abstracto.

Según la teoría dialectico-materialista, el conocimiento concreto es la primera etapa donde el hombre, inmerso en la práctica, logra el reflejo del mundo, a través de sus sensaciones, percepciones y representaciones. El conocimiento abstracto o racional es aquel mediante el cual el hombre realiza los procesos lógicos del pensamiento, tales como: el análisis, la síntesis, la generalización, como también arriba a conclusiones sobre la esencia y los vínculos internos de los objetos y fenómenos (Martínez, 2007, p. 84).

Toda enseñanza-aprendizaje de cualquier hecho, fenómeno o asunto debe partir del conocimiento directo y concreto del mismo, para llegar a través de distintos procesos mentales de análisis, síntesis, generalización, hipótesis, etc, a mayores niveles de profundización. De ahí la importancia que tiene la presentación del hecho o fenómeno en sí, o en su lugar, la representación del fenómeno a través de imágenes, modelos, u otras formas, y no solamente a través del lenguaje (Antich, 1986, p.52).

Para el proceso docente de las lenguas extranjeras, la consideración del principio del carácter audiovisual de la enseñanza: unión de lo concreto y lo abstracto, resulta de gran importancia si se considera que en "la enseñanza de una lengua extranjera con ayuda visual, se forman relaciones asociativas entre el objeto y el mundo material, y su representación con la palabra y el concepto" (González, 2009, p. 12).

El papel de estos en el marco del proceso docente rebasa la simple función de ser el contacto 
sensorial, el enfrentamiento concreto con la naturaleza [...] lejos de eso, el papel de los medios está en proporcionar verdaderamente el puente o vínculo entre estas percepciones concretas y el proceso lógico del pensamiento (Castro, 1990, p. 49).

En resumen, el carácter audiovisual de la enseñanza contextualizado al proceso docente de las lenguas extranjeras nos indica la necesidad de crear condiciones para un mayor acercamiento del sujeto que aprende a la realidad comunicativa en el contexto del salón de clases. De ahí la importancia en cuanto al uso de diversos medios audiovisuales que garanticen una profunda interiorización de la realidad que se presenta y una adecuada simulación en condiciones muy cercanas a la realidad. Todo ello habrá de redundar no solo en la efectividad del proceso, sino también en la eficiencia del mismo en cuanto a tiempo y uso racional del esfuerzo mental del estudiante. En el presente, no se concibe un proceso docente de las lenguas extranjeras ajeno al uso de medios de enseńanza tradicionales, como tampoco alejado de las nuevas propuestas que resultan del desarrollo tecnológico actual.

\section{Conclusiones}

A través del presente artículo se ha tratado de responder al interrogante: Didáctica general y específicas ¿autonomía, dependencia, coexistencia o complementación? El análisis se ha basado fundamentalmente en los postulados de la pedagogía y la didáctica cubana, así como en la experiencia emanada del proceso docente de las lenguas extranjeras en el ámbito nacional, pero matizada con los supuestos de especialistas internacionales, contextualizados a la realidad de sistema educativo cubano.

Desde el enfoque que asumimos, podemos alegar que a pesar de no declarar a la didáctica general y las particulares como iguales, a partir de las particularidades de sus objetos de estudio, resulta evidente y necesario el vínculo que entre ellas se establece. La didáctica general revela las regularidades comunes que se manifiestan en los diferentes procesos de enseñanza y aprendizaje y, sobre esa base, constituye un fundamento teórico y metodológico de gran relevancia para el trabajo didáctico específico. En esta relación, las didácticas específicas se convierten en fuentes de la didáctica general, ya que aportan información inestimable para la dirección efectiva de los diversos procesos docentes. De tal manera, se fundamenta la relación entre ambas didácticas. Entre ellas se establece una contradicción a partir de sus especificidades, lo que como condición de desarrollo conduce a la consumación de un objetivo común: la preparación del hombre para la vida.

Lo anteriormente expuesto se expresa de manera clara en la relación que se establece entre la didáctica general y la específica de las lenguas extranjeras en el contexto educacional cubano. Tal y como hemos demostrado a lo largo de nuestro análisis, la didáctica general contribuye de manera significativa a regular la función del profesor de organizar y dirigir de forma científica y pertinente el proceso docente de las lenguas extranjeras de acuerdo con el encargo social y personal que se establece.

Sirva el presente artículo de fuente para la reflexión acerca del tema planteado, que nos permita asumir una actitud de cambio respecto a nuestro quehacer docente, sobre la base de una concepción flexible, que nos permita contextualizar los aportes de otras ciencias afines de acuerdo con las especificidades de nuestras materias. Rómpase, pues, con conceptos estereotipados y asúmase una actitud dialéctica, de cambio, conducente a un desarrollo significativo en los individuos que participan en nuestros procesos docentes e integran nuestras sociedades.

\section{Referencias}

Agramonte, A., Vázquez, F. R., \& Iznaga, V. I. (2012). Reflexiones necesarias para aplicar los principios de la enseñanza en la educación de postgrado en enfermería. Revista cubana de enfermería, 28(1), 13-14.

Álvarez, Z. C. (1995). La escuela en la vida. La Habana: Colección Educación y Desarrollo.

Antich, R. (1986). Metodología de la enseñanza de lenguas extranjeras. La Habana: Pueblo y Educación. 


\section{MARTÍNEZ-LINARES}

Didáctica general y específica de las Lenguas Extranjeras en Cuba, ¿̇autonomía, dependencia...

Camilloni, A. (2007). El saber didáctico. Cap. 2 Didáctica general y didácticas especificas. Argentina: Instituto de Educación Superior Amadeo Sirolli.

Castro, V. G. (1990). Teoría y práctica de los medios de enseñanza. La Habana: Pueblo y Educación.

Corona, C. D. (1988). El perfeccionamiento de la enseñanza de la lengua extranjera a estudiantes no filólogos en la educación superior cubana. La Habana: Universidad de la Habana.

Danilov, M., \& Skatkin, M. (1980). Didáctica de la escuela media. La Habana: Libros para la Educación.

Font, S. (2006). Metodología para la asignatura inglés en la secundaria básica desde una concepción problemática del enfoque comunicativo. La Habana: Universidad de las Ciencias Pedagógicas Enrique José Varona.

Góngora, A. P. (2014a). El desarrollo de habilidades de comunicación oral en la formación semipresencial de profesores de inglés. La Habana: Universidad de Ciencias Pedagógicas Enrique José Varona.

Góngora, A. P. (2014b). Concepciones didácticas de la enseñanza de lenguas extranjeras apoyadas en la creación de software educativos. La Habana: Universidad de Ciencias Pedagógicas Enrique José Varona.

González, R. (2009). La clase de lengua extranjera, teoría y práctica. La Habana: Pueblo y Educación.
Jiménez, A. \& Falcón, D. (2006). Los principios didácticos, guía segura del profesor. Revista Pedagógica Universitaria 11(3), 15-44.

Konstantinov, N., Medinski, E., \& Shabaeva, M. (1977). Historia de la Pedagogía. La Habana: Pueblo y Educación.

Klimberg, L. (1972). Introducción a la didáctica general. La Habana: Pueblo y Educación.

Labarrere, G., \& Valdivia, P. (2009). Pedagogía. La Habana: Pueblo y Educación.

Martí, J. (1884). Obras Completas. Vol. 3. La Habana. Buenos Aires: Centro de Estudios Martianos.

Martínez, C., \& Novo. T. (2007). El currículo de la escuela contemporánea. Panamá: Meduca.

Martínez, C. (2017). Incidencia de la didáctica general en el proceso docente de las lenguas extranjeras en Cuba. Íkala. Revista de Lenguaje y Cultura, 22(1), 71-86.

Pulido, A. D. (2005). Didáctica desarrolladora de lenguas extranjeras. Pinar del Río, Cuba: Instituto Superior Pedagógico de Pinar del Río Rafael María de Mendive.

Richards. J. C. (2013). Curriculum Approaches in Language Teaching: Australia: University of Sydney.

\section{CÓMO CITAR:}

Martínez-Linares, C. (2021). Didáctica general y específica de las Lenguas Extranjeras en Cuba, ¿Autonomía, dependencia, coexistencia o complementación? RECIE. Revista Caribeña de Investigación Educativa, 5(1), 148-161. https://doi.org/10.32541/ recie.2021.v5i1.pp148-161 